\title{
“EFECTOS DE USO DEL SOFTWARE MATEMÁTICO MATLAB SOBRE EL RENDIMIENTO ACADÉMICO DE UN GRUPO DE ESTUDIANTES REPITENTES DE MATEMÁTICA BÁSICA"
}

\author{
"EFFECTS OF USE OF MATLAB MATHEMATICAL SOFTWARE ON THE \\ ACADEMIC PERFORMANCE OF A GROUP OF REPEATING STUDENTS \\ OF BASIC MATHEMATICS" \\ (iD) Franklin Surichaqui Gutierrez ${ }^{*}$, (D) Felisícimo German Ramírez Rosales ${ }^{1}$, (D) Melvy Rosario \\ Mercado Boza ${ }^{1}$
}

Fsurichaquigutierrez@gmail.com; ramirezfeli7@hotmail.com; mmercadoboza@gmail.com ${ }^{1}$ Universidad Nacional de Huancavelica, Huancavelica, Perú

*Correspondencia: Franklin Surichaqui Gutierrez. Email: Fsurichaquigutierrez@gmail.com

Recibido: 24.03.2020 | Aprobado: 09.04.2020

\section{RESUMEN}

En la formación de estudiantes Universitarios, se ha evaluado el efecto del uso del software Matlab sobre el rendimiento académico en un grupo de estudiantes repitentes de matemática básica, investigación de tipo cuasi experimental, cuantitativo con un diseño de investigación pre-experimental de pre prueba - post prueba con un solo grupo; se tuvo una muestra de 48 estudiantes repitientes distribuidos en un solo grupo, se usó evaluó rendimientos académicos individuales en la escala de 0 al 20; se llega a la conclusión que el uso del software matemático Matlab influye positivamente en el rendimiento académico de un grupo de estudiantes repitentes.

Palabras clave: Matlab, rendimiento académico, TIC, universitario.

\begin{abstract}
In the training of University students, the effect of the use of Matlab software on academic performance has been evaluated in a group of repeating students of basic mathematics, quasi-experimental, quantitative research with a pre-experimental pretest research design - post test with a single group; There was a sample of 48 repeating students distributed in a single group, using individual academic performance on the scale of 0 to 20; It is concluded that the use of Matlab mathematical software positively influences the academic performance of a group of repeating students.
\end{abstract}

Keywords: Matlab, academic performance, NTIC, university. 


\section{INTRODUCCIÓN}

La formación universitaria va cambiando de acorde con los avances tecnológicos que se promueve en el mundo, tal es así el uso de las nuevas tecnologías de la información y comunicaciones ha hecho de que en las aulas universitarias también se puedan usar estas herramientas con la finalidad de generar mayor rendimiento académico durante la formación profesional de los estudiantes de ingeniería.

Es mas muchos autores coinciden de que el uso de las Tecnologías de la Información y Comunicaciones (TIC) son favorables para mejorar el rendimiento académico de los estudiantes como lo citan (Valencia, Esteban, \& Ramirez, 2019, pág. 250).

Esta investigación en donde se usan la TIC para favorecer el desarrollo de los estudiantes especialmente en el área de comunicación haciendo uso de la laptop XO tratando de relacionar el uso del laptop XO en desarrollar competencias en el área de comunicación como lo cita (Gutierrez, 2009, pág. 96).

De la investigación anterior podemos aprender que los estudiantes a pesar de que son considerados como nativos digitales aun así necesitan saber cuáles son los beneficios del uso de las TIC en el proceso de su aprendizaje dándoos a conocer que también una parte importante de la responsabilidad recae en los docentes quienes debieran de mostrar los beneficios del uso de las TIC a los estudiantes (Valencia, Esteban, \& Ramirez, 2019).

Por otra parte (Correa, 2016, pág. 122) concluye que hay evidencia científica para demostrar la relación que existe entre el uso de las tecnologías de la información y comunicación tenga una relación directa y positiva con el desempeño docente, de esta investigación no solo podemos concluir que ayuda a los estudiantes, sino que también hace que los docentes tengan mayor desempeño docente.

Además, se ha encontrado que el uso de algunas TIC ayuda bastante el proceso de enseñanza - aprendizaje y el rendimiento académico de la matemática de estudiantes como lo afirma (Encalada, 2018, pág. 134).

Dávila (2007) afirma que el uso de tecnologías digitales aplicado en la enseñanza de las matemáticas si mejora significativamente, aunque estadísticamente no sea significativa,

Básicamente en este trabajo se estudia acerca de los factores relacionados al uso de las tecnologías educativas por parte de los docentes de la facultad de Ingeniería Minas Civil Ambiental de la Universidad Nacional de Huancavelica durante el semestre académico 2008-I con la intención de aportar elementos metodológicos de enseñanza y aprendizaje para orientar una posible solución del problema representado por el alto índice de aplazados en la asignatura de formación general de Matemática Básica en este centro de superior de estudios.

Por lo que se plantea el siguiente problema de investigación: ¿Qué efectos tendrá el uso del software matemático Matlab 7.0? sobre el rendimiento académico de los estudiantes repitentes de matemática básica de los alumnos de la FIMCA de la Universidad Nacional de Huancavelica? 
La hipótesis formulada: El uso del software matlab 7.0 mejora el rendimiento académico de los estudiantes repitentes de matemática básica de los alumnos de la FIMCA de la Universidad Nacional de Huancavelica

El objetivo general: Mejorar los procesos de enseñanza y aprendizaje utilizando el software matemático matlab 7.0 en los estudiantes repitentes de matemática básica de la FIMCA de la Universidad Nacional de Huancavelica. Objetivos específicos: a). Estudiar y Organizar los tópicos de enseñanza de las definiciones de matrices y propiedades. b). Combinar la parte teórica y práctica aplicando el software Del Matlab 7.0, para encontrar la solución de matrices que resuelva las incógnitas propuestas.

\section{MATERIALES Y MÉTODOS}

La investigación consistió en una manipulación de la variable independiente software matlab. Con este propósito, se dio uso intensivo al servicio de del software matlab en actividades propias del proceso instruccional de Matemáticas Básicas. Consecuentemente, se observaron los efectos de esas manipulaciones en la variable dependiente rendimiento académico de los estudiantes repitientes de Matemáticas Básicas. La diferencia crucial con el mismo grupo, también tomado como grupo control, fue que en todas las ocasiones anteriores en que cursaron Matemática Básica se les enseñó con métodos tradicionales, por exposiciones magistrales del profesor sin uso de tecnología digital. Para controlar la variable independiente, y prevenir la intervención e influencia de la variable conocimientos previos, se aplicó una prueba exploratoria de conocimientos sobre el programa de Matemáticas Básicas, al inicio del experimento. La prueba tuvo una duración de tres horas, y se aplicó presencialmente en la semana inicial de actividades, en horario extra. El análisis de los resultados de esta prueba demostró que la variable conocimientos previos no comprometió la validez interna del experimento. Los estudiantes participantes recibieron 1 hora semanal de entrenamiento en el uso de software de matlab, ya que los estudiantes conocían poco de este programa solo se les impartió conocimientos básicos de operaciones de matrices, todo esto también quizás se llevó en cuenta por los horarios ajustados del primer ciclo. Nosotros los investigadores facilitamos ese entrenamiento en los laboratorios de computación de la Universidad Nacional de Huancavelica y en el aula de clases, durante las cuatro primeras semanas de actividades del curso usando el software matlab, los estudiantes resolvieron problemas para reforzar teorías e ilustrar conceptos de Matrices y operaciones de ellas mismas, ya que estos temas son de vital importancia para temas de suma interés en los cursos superiores.

En cada trabajo, se propuso al menos un problema que debió ser resuelto analíticamente. Así, se crearon situaciones de aprendizaje para que los estudiantes fijaran conceptos y desarrollaran habilidades intelectuales para resolver problemas, además de presentar sus soluciones gráficas. Las soluciones gráficas a los problemas planteados fueron entregadas como archivos anexos a través del servicio de correo electrónico. Para los desarrollos teóricos, los estudiantes tuvieron la libertad de entregar las soluciones en forma manuscrita o digitalizada. De los trabajos asignados, fueron individuales. Los investigadores nos reservamos el derecho a interrogar en privado a los estudiantes sobre los trabajos entregados para asignarles la calificación correspondiente, ya fueron 
individuales. Estos trabajos tuvieron una ponderación del 25\% sobre la calificación final sumativa del curso. El desarrollo de conceptos de teoremas y definiciones básicas de matrices y sus aplicaciones, con mediación del software Matlab, fue el objetivo principal de la metodología de enseña luego de todo esto podemos decir que el primer hallazgo demostró que el rendimiento académico de los estudiantes que repitieron Matemática Básica con el uso del software Matlab mejoro significativamente ya que el grupo de estudio aprobaron el 100\%

Los métodos que se usaron son : análisis, síntesis, deducciones, inducciones, descripciones y estadístico donde se utilizó un diseño pre-experimental de pre-prueba post-prueba con un solo grupo para realizar un análisis estadístico de los puntajes obtenidos en dichas pruebas, este diseño consiste en que a un grupo $(G)$ se le aplica una prueba previa $\left(\mathrm{O}_{1}\right)$ al estímulo o tratamiento experimental; después se le administra el tratamiento $(\mathrm{X})$ y finalmente se le aplica una prueba posterior $\left(\mathrm{O}_{2}\right)$ al tratamiento.

Donde en el manejo del experimento se consideraron dos variables: la variable independiente constituida por el método del uso del Software Matemático matlab y variable dependiente constituida por rendimiento académico de los estudiantes repitentes de matemática básica

\section{RESULTADOS}

Primero mostraremos el cuadro de resultados del pre test y post test de las evaluaciones mencionadas durante este trabajo.

\section{Tabla 1}

Resultados del pre y post test

\begin{tabular}{lllll}
\hline $\mathbf{N}^{\mathbf{0}}$ & $\begin{array}{l}\text { variable } \\
\text { independiente }\end{array}$ & $\begin{array}{l}\text { variable } \\
\text { dependiente }\end{array}$ & $\begin{array}{l}\text { variable } \\
\text { independiente }\end{array}$ & $\begin{array}{l}\text { variable } \\
\text { dependiente }\end{array}$ \\
\hline 1 & 12 & 9 & 14 & 16 \\
2 & 13 & 7 & 15 & 16 \\
3 & 12 & 9 & 12 & 13 \\
4 & 11 & 11 & 11 & 12 \\
5 & 10 & 7 & 17 & 16 \\
6 & 11 & 8 & 13 & 14 \\
7 & 10 & 8 & 14 & 15 \\
8 & 9 & 7 & 15 & 15 \\
9 & 12 & 10 & 14 & 16 \\
10 & 11 & 12 & 12 & 13 \\
11 & 8 & 7 & 15 & 16 \\
12 & 11 & 9 & 14 & 16 \\
13 & 11 & 7 & 13 & 14 \\
14 & 12 & 10 & 16 & 17 \\
15 & 14 & 11 & 19 & 18 \\
16 & 12 & 11 & 15 & 16 \\
17 & 11 & 10 & 14 & 14 \\
18 & 13 & 12 & 12 & 13 \\
19 & 10 & 11 & 15 & 16 \\
20 & 9 & 8 & 18 & 16 \\
21 & 11 & 9 & 13 & 14 \\
22 & 11 & 10 & & \\
\hline
\end{tabular}




\begin{tabular}{lllll}
\hline 23 & 13 & 11 & 12 & 13 \\
24 & 10 & 12 & 17 & 18 \\
25 & 12 & 11 & 16 & 15 \\
26 & 9 & 8 & 15 & 15 \\
27 & 11 & 11 & 14 & 13 \\
28 & 13 & 12 & 13 & 15 \\
29 & 10 & 10 & 15 & 16 \\
30 & 12 & 11 & 11 & 12 \\
31 & 12 & 12 & 12 & 13 \\
32 & 11 & 12 & 15 & 14 \\
33 & 9 & 10 & 16 & 16 \\
34 & 10 & 11 & 19 & 18 \\
35 & 10 & 12 & 17 & 16 \\
\hline
\end{tabular}

Fuente: Elaboración propia.

Luego de ello pasamos a hacer el análisis de correlación, es decir los datos obtenidos nos permiten hacer un análisis correlacional que busca determinar el grado de correlación que existe entre la variable independiente y la variable dependiente tomando en cuenta:

Ingresaremos los datos de la post prueba usando el programa SPSS V.15 para el análisis de la misma:

\section{Tabla 2}

Datos de post prueba de las variables independiente y dependiente

\begin{tabular}{|c|c|c|}
\hline $\mathbf{N}^{\mathbf{o}}$ & $\begin{array}{l}\text { variable } \\
\text { independiente }\end{array}$ & $\begin{array}{l}\text { variable } \\
\text { dependiente }\end{array}$ \\
\hline 1 & 14 & 16 \\
\hline 2 & 15 & 16 \\
\hline 3 & 12 & 13 \\
\hline 4 & 11 & 12 \\
\hline 5 & 17 & 16 \\
\hline 6 & 13 & 14 \\
\hline 7 & 14 & 15 \\
\hline 8 & 15 & 15 \\
\hline 9 & 14 & 16 \\
\hline 10 & 12 & 13 \\
\hline 11 & 15 & 16 \\
\hline 12 & 14 & 16 \\
\hline 13 & 13 & 14 \\
\hline 14 & 16 & 17 \\
\hline 15 & 19 & 18 \\
\hline 16 & 15 & 16 \\
\hline 17 & 14 & 14 \\
\hline 18 & 12 & 13 \\
\hline 19 & 15 & 16 \\
\hline 20 & 18 & 17 \\
\hline 21 & 13 & 14 \\
\hline 22 & 14 & 15 \\
\hline 23 & 12 & 13 \\
\hline 24 & 17 & 18 \\
\hline
\end{tabular}




\begin{tabular}{lll}
\hline 25 & 16 & 15 \\
26 & 15 & 15 \\
27 & 14 & 13 \\
28 & 13 & 15 \\
29 & 15 & 16 \\
30 & 11 & 12 \\
31 & 12 & 13 \\
32 & 15 & 14 \\
33 & 16 & 16 \\
34 & 19 & 18 \\
35 & 17 & 16 \\
\hline
\end{tabular}

Fuente: Elaboración propia.

Luego primero veremos el grado de dispersión que existe de relación entre la variable dependiente e independiente

\section{Figura 1}

Grado de dispersión de los datos obtenidos

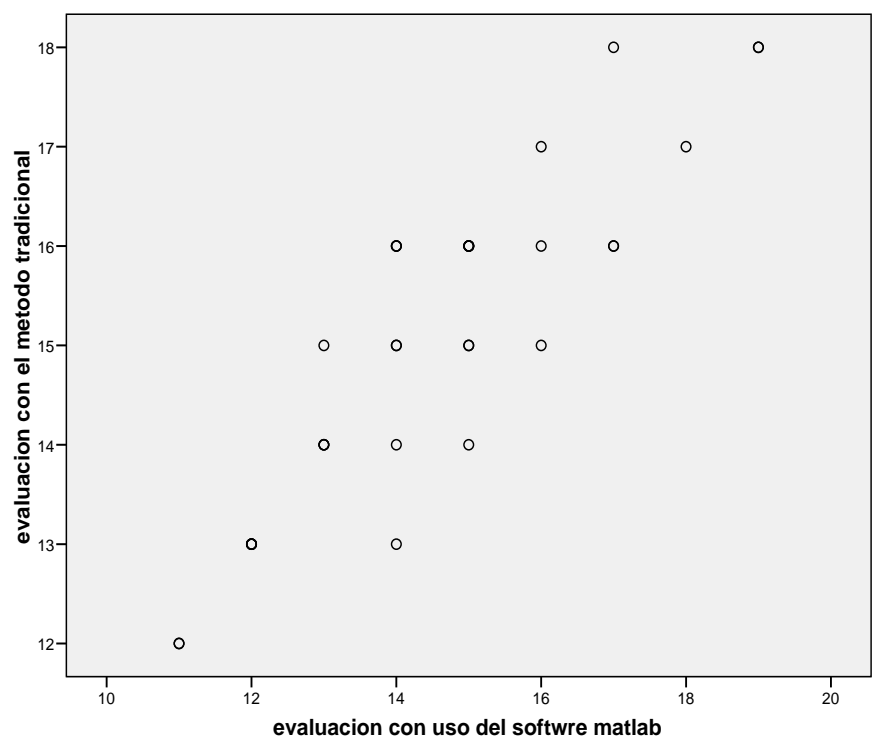

Fuente: Elaboración propia.

\section{Luego haciendo:}

$\checkmark \quad$ Realizando una regresión Lineal obtenemos:

\section{Tabla 3}

Coeficientes(a)

\begin{tabular}{llllll}
\hline Modelo & \multicolumn{2}{l}{$\begin{array}{l}\text { Coeficientes no } \\
\text { estandarizados }\end{array}$} & $\begin{array}{l}\text { Coeficientes } \\
\text { estandarizados }\end{array}$ & t & Sig. \\
\cline { 2 - 6 } & $\mathbf{B}$ & Error típ. & Beta & B & Error típ. \\
\hline $\begin{array}{l}\text { (Constante) } \\
\begin{array}{l}\text { evaluación con uso } \\
\text { del software matlab }\end{array}\end{array}$ & 4,810 &, 939 & & 5,122 &, 000 \\
\hline
\end{tabular}


a. Variable dependiente: evaluación con el método tradicional

El coeficiente de correlación es: $r=0.886$. Explica que las notas del examen con el software matlab y las notas del examen con el método tradicional final tienen una correlación positiva. Lo que nos indica que hay una correlación lineal positiva y eso se reafirma con la gráfica anterior.

Entonces para obtener el resultado buscado hallamos el coeficiente de determinación:

b. Variables predictoras: (Constante), evaluación con uso del SOFTWARE MATLAB

Donde el coeficiente de determinación es: 0,785 = 78.5\%

\section{Tabla 4}

Resumen del modelo

\begin{tabular}{|c|c|c|c|c|c|c|c|c|c|}
\hline \multirow{2}{*}{$\begin{array}{c}\text { Modelo } \\
1\end{array}$} & \multirow{2}{*}{$\begin{array}{l}\mathbf{R} \\
\text { Cambio } \\
\text { en R } \\
\text { cuadrado }\end{array}$} & \multirow{2}{*}{$\begin{array}{l}\text { R cuadrado } \\
\text { Cambio en F }\end{array}$} & \multirow{2}{*}{$\begin{array}{l}\text { R cuadrado } \\
\text { corregida }\end{array}$} & \multirow{2}{*}{$\begin{array}{l}\begin{array}{l}\text { Error típ. } \\
\text { de la } \\
\text { estimación }\end{array} \\
\text { g12 }\end{array}$} & \multicolumn{5}{|c|}{ Estadísticos de cambio } \\
\hline & & & & & $\begin{array}{l}\text { Sig. del } \\
\text { cambio } \\
\text { en F }\end{array}$ & $\begin{array}{l}\text { Cambio } \\
\text { en R } \\
\text { cuadrado }\end{array}$ & $\begin{array}{l}\text { Cambio } \\
\text { en F }\end{array}$ & gl1 & gl2 \\
\hline & ,886(a) & ,785 & (779 & ,778 & ,785 & 120,779 & 1 & 33 & ,000 \\
\hline
\end{tabular}

\section{CONCLUSIONES}

La hipótesis de los investigadores sobre el mejoramiento del rendimiento académico se confirmó. Estos resultados también confirmaron los hallazgos de numerosos estudios sobre el mismo problema (Davila, 2007) (Encalada, 2018) (Valencia, Esteban, \& Ramirez, 2019) De modo el uso de software para mejorar el rendimiento académico y el grado de aprovechamiento de los estudiantes en el aprendizaje de las matemáticas queda evidenciada y por ello este estudio aporta elementos para que se fomente el uso del software matemático del matlab en la enseñanza y aprendizaje de Matemáticas Básicas en estudiantes universitarios de las carreras de ingeniería.

Además podemos concluir que el uso de herramientas computacionales o de las TIC, ayudaran básicamente en la mejor comprensión de las matemáticas y de otros área en los estudiantes asi podrían tener mayores herramientas en su futuro profesional y por otra parte también los maestros son beneficiados ya que vimos que existe una relación directa y positiva en la mejora del desempeño de los maestros y esto nos conlleva a definir nuestra tesis sobre el mejoramiento del rendimiento académico usando el software Matlab en el aprendizaje de las matemáticas en estudiantes universitarios de la carrera de ingeniería.

\section{REFERENCIAS BIBLIOGRÁFICAS}

Correa, Q. (2016). Uso de las tecnologias de la informción y comunicación y el desempeño de los docentes de educación básica secundaria en la institucion educativa Jorge Eliacer Gaitan Tota - Boyacá 2016. Tesis Magister, Lima.

Davila, A. (2007). Efectos de algunas tecnologías digitales sobre el rendimiento académico en matemáticas. Redalyc, 22-36. 
Encalada, I. (2018). Uso del software educativo cuadernia en el proceso de enseñanza aprendizaje y en el rendimiento académico de la matemática de los estudiantes del 5 to año de secundaria de la Institución Educativa $N^{\circ} 5143$ Escuela de Talentos - Callao. Callao.

Gutierrez, G. (2009). Uso de las computadoras portatiles XO en el desarrollo de los componentes del área de Comunicación Integral en los alumnos del sexto grado de la I.E. N 30115 del centro poblado Chucupata en Junín. Lima.

Valencia, L., Esteban, T., \& Ramirez, F. (2019). Programa Edusofr y desempeño docente en las instituciones educativas públicas del nivel secundario. Puriq, 250.

\section{CITAR COMO:}

Surichaqui Gutierrez, F., Ramírez Rosales, F. G., \& Mercado Boza, M. R. (2020). Efectos de uso del software matemático Matlab sobre el rendimiento académico de un grupo de estudiantes repitentes de matemática básica. Puriq, 2(2), 119-126. https://doi.org/10.37073/puriq.2.2.78 